\title{
PPM de flúor rotulado y analizado en pastas dentales pediátricas comercializadas en Lima-Perú
}

\author{
PPM of labeled fluorine and analyzed fluorine in pediatric toothpaste commercialized in Lima-Peru
}

Ofelia Córdova-López ${ }^{1, a}$; Rocío Victoria Hermoza-Moquillaza ${ }^{1, b, c}$; Darwin Yanac Calero ${ }^{1, e}$, César Arellano-Sacramento ${ }^{2, a, d}$.

\section{RESUMEN}

Objetivos: Determinar la concentración de ppm de flúor en pastas dentales para niños que se comercializan en Lima Perú en el año 2017. Material y métodos: Es un estudio observacional, descriptivo, transversal. Fueron recolectadas 48 pastas dentales pediátricas vendidas en establecimientos farmacéuticos (farmacias y boticas) de Lima. Se empleó las pruebas de $t$ de Student y Wilcoxon para una sola muestra. Resultados: La diferencia entre la concentración de flúor según rotulado y encontrado en Colgate de 1100 ppm fue -119,8 ppm (mediana), Oral $\mathrm{B}$ de 500 ppm fue de -57,05 ppm (mediana). La diferencia entre la concentración de flúor según rotulado y lo encontrado en base al promedio fue Colgate de 500 ppm: -34,2 ppm, Farmadent de 452 ppm: -36,27 ppm, Dentito de $550 \mathrm{ppm}:-520,1 \mathrm{ppm}$, Aqua fresh de 500 ppm: +41,67 ppm, Vitis de $1000 \mathrm{ppm}:+141,85 \mathrm{ppm}$, Aqua Fresh de 1150 ppm: +112.82 ppm; de entre los mencionados sólo Aqua Fresh de 500 ppm fue no significativo. Con respecto al $\mathrm{pH}$ de las pastas dentales, los promedios fueron 5,$92 ; 5,5 ; 5,5 ; 6 ; 6 ; 5,5 ; 5,5 ; 6$, respectivamente. Conclusiones: Cinco tipos de pastas dentales tienen concentraciones menores al rotulado, y tres tipos de pastas dentales tienen concentraciones mayores al rotulado (de las cuales una no fue significativa).

PALABRAS ClAVES: Flúor, pH, niños, pastas dentales, Perú.

\section{SUMMARY}

Objective: To determine the concentration of fluorine ppm in toothpastes for children that are commercialized in Lima Peru in the year 2017. Material y Methods: It is an observational, descriptive, cross-sectional study. 48 pediatric toothpastes sold in pharmaceutical establishments (pharmacies and drugstores) in Lima were collected. The Student $t$ and Wilcoxon $t$ tests were used for a single sample. Results: The difference between the concentration of fluorine as labeled and found in Colgate of $1100 \mathrm{ppm}$ was $-119.8 \mathrm{ppm}$ (median), Oral B of $500 \mathrm{ppm}$ was $-57.05 \mathrm{ppm}$ (median). The difference between the concentration of fluorine according to labeling and that

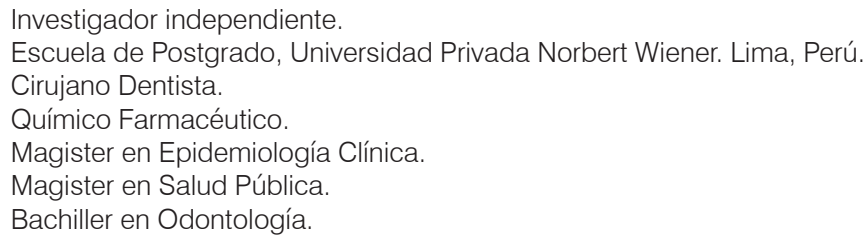


found based on the average was Colgate of 500 ppm: -34.2 ppm, Farmadent of 452 ppm: -36.27 ppm, Dentito of $550 \mathrm{ppm}:-520.1 \mathrm{ppm}$, Aqua fresh of $500 \mathrm{ppm}:+41.67 \mathrm{ppm}$, Vitis of $1000 \mathrm{ppm}:+141.85 \mathrm{ppm}$, Aqua Fresh of 1150 ppm: $+112.82 \mathrm{ppm}$; among those mentioned only Aqua Fresh 500 ppm was not significant. With respect to the $\mathrm{pH}$ of toothpastes, the averages were $5.92 ; 5.5 ; 5.5 ; 6 ; 6 ; 5.5 ; 5.5 ; 6$, respectively. Conclusions: Five types of toothpaste have lower concentrations than labeling and three types of toothpastes have concentrations higher than the labeling (of which one was not significant).

\section{KEY WORDS: Fluoride, pH, children, toothpaste, Peru.}

\section{INTRODUCCIÓN}

La incorporación de flúor en las pastas dentales ha logrado la reducción de caries dental (1). En el Perú, el $85 \%$ de menores de 11 años y el $76 \%$ de entre 3 a 5 años tienen caries dental; siendo esta situación, consecuencia de una inadecuada higiene bucal (2).

El Ministerio de Salud del Perú (MINSA) menciona que las cremas dentales (refriéndose a la pasta dental) con una concentración de fluoruro que sobrepase 1 100 ppm (partes por millón)., serán aplicadas a niños mayores de 6 años y, en caso que el niño sea menor de 6 años, la pasta deberá tener una concentración de fluoruro de 250 a $550 \mathrm{ppm}$ (3). Sin embargo, una guía de práctica clínica aprobada en el Perú aconseja que sean aplicadas aquellas pastas dentales con un mínimo de 1000 ppm de flúor desde que aparece el primer diente deciduo (2).

En nuestro país, algunas pastas dentales para menores de edad de venta en el mercado, no tienen efecto preventivo contra la caries dental, al no tener flúor o presentarlo en cantidades insuficientes (2). Por ello, la elección de la forma de aplicación de fluoruros en la población, debe surgir del uso de herramientas técnicas (4).

La cantidad que indica el rotulado de la pasta dental, puede diferir con la cantidad exacta de flúor, pues es un intervalo aproximado; cada fabricante determina su propio rango. Esta cantidad exacta, se podría calcular individualmente como la diferencia entre lo Rotulado (según lo que menciona el producto) y lo Encontrado (según el análisis de laboratorio del producto ya en el mercado); en el Perú es la Dirección General de Medicamentos y Drogas (DIGEMID), la autoridad encargada de la inspección de este tipo de productos (5).

En Perú la Dirección General de Medicamentos y
Drogas (DIGEMID) es la autoridad a quien le corresponde la inspección de los productos farmacéuticos, dispositivos médicos y productos sanitarios para asegurar que cumplan con las especificaciones y técnicas analíticas de los productos que mencionan en sus registros correspondientes. Por lo que le corresponde la inspección de los productos analizados (5).

Las pastas dentales con flúor son el medio más utilizado para prevenir la caries dental (4), por ello, el valor de sus diversos elementos, no deberían estar fuera de los rangos de sus especificaciones técnicas; pues al presentar valores inferiores pueden llevar a una baja eficacia y valores mayores (probable efecto de fluorosis dental o intoxicación con flúor si se utiliza en forma diferente a lo recomendado por el dentista).

El consumidor considera que las cantidades que contienen los medicamentos y productos sanitarios presentan el valor mencionado en el rotulado, desconociendo que su variación está regida por valores aprobados por cada país, cambios físicos (almacenaje, transporte), manipulación por parte del consumidor, etcétera.

La sociedad civil debe estar alerta ante las posibles variaciones en los productos que nos ofrecen el mercado.

El objetivo del estudio fue determinar la concentración de ppm de flúor en pastas dentales para niños que se comercializan en Lima, Perú.

\section{MATERIAL Y MÉTODOS}

Fue un estudio observacional, descriptivo, transversal. La unidad de estudio fueron tubos de pastas dentales para niños comercializadas en boticas y farmacias de Lima en el año 2017.

Para el estudio, "marca de pasta dental" fue basado en el concepto de INDECOPI de "marca de producto" un signo empleado para distinguir y diferenciar el 
producto en el mercado de otros similares. Diferenciándolo de "tipo de pasta" que puede ser por el sabor, color, etcétera (6).

Se eligieron seis marcas de pasta dental pediátrica, las mismas que se utilizaron en un estudio nacional (7), añadiendo para el presente estudio dos tipos más; siendo al final ocho grupos. Se utilizó la fórmula para comparación de medias, por lo que ante un del 95\% de confianza, poder de $80 \%$ y un $\mathrm{DE} / \mathrm{d}=0,60$ (7) se obtuvo: 5,64. Por lo que se requirió seis tubos de pastas dentales por grupo (48 unidades).

Las pastas dentales utilizadas fueron elegidas por conveniencia. Los criterios de inclusión fueron que las pastas dentales fluoradas expendidas en Lima considerados para uso pediátrico, mencionen la concentración de flúor en el rotulado y en los ingredientes. Los criterios de exclusión fueron que las pastas dentales estén perforadas, deterioradas o con empaque no sellada, vendidos en lugares informales, con fechas de vencimiento corta (mayor de 6 meses a su expiración).

Se procedió a adquirir seis muestras de cada tipo de pasta dental en diferentes establecimientos farmacéuticos, elegidos por conveniencia, siendo un total de 48 unidades, considerando los criterios de inclusión. No se descartó ninguna de las pastas dentales obtenidas. El mínimo valor probable de flúor en el rotulado, según las pastas dentales elegidas fue de 452 de ppm de flúor, siendo el máximo 1150 de ppm de flúor.

La concentración mínima posible de ppm de flúor que podría ser encontrado en el análisis sería cero; el límite no puede ser calculado a priori.

El cálculo de la concentración de flúor y $\mathrm{pH}$ en las pastas dentales fue realizado en el Laboratorio de la Facultad de Farmacia y Bioquímica de la Universidad Nacional de Trujillo; en la cual se han llevado a cabo investigaciones similares (7).

El analista del laboratorio en todo momento conocía el tipo de pasta a analizar. La industria farmacéutica utiliza tubos colapsibles en las pastas dentales, los rotulados están impresos en forma directa no mediante una etiqueta desprendible, siendo además estos tubos característicos e identificables por marca. Asimismo, las características de las cremas dentales (color y olor) son propias de cada laboratorio; siendo con esto identificable por marca. Por todo esto, no se consideró el análisis en ciego para el analista de laboratorio.

El volumen de un tubo de pasta dental fue colocado en un mortero de porcelana, homogenizado y pesado, resultando alrededor de 2,0 y 3,0 g de muestra, el cual fue diluido con agua desionizada a $100 \mathrm{~mL}$. De esto, se midió $5 \mathrm{~mL}$ de muestra en estudio y 5 $\mathrm{mL}$ de TISAB II (solución para uso con electrodos selectivos de ión fluoruro) dentro de un envase de polietileno de $25 \mathrm{~mL}$; se agitó completamente y esperó hasta que la lectura se estabilice, aceptándose luego el valor. Este procedimiento se realizó por triplicado, para obtener un valor preciso. Se usó el fluorímetro de lectura directa 720 A (Electrodo ISE), el cual se basa en la diferencia de potencial entre el contacto del electro con el ión estudiado y un electrodo de referencia. Esta es la metodología de laboratorio utilizada en al análisis de reconocimiento de flúor.

Los valores conseguidos de fluoruro fueron declarados en partes por millón (ppm).

Los valores son dichos en términos de valores centrales y valores dispersos como la desviación estándar. Los análisis se desarollaron por triplicado en las 48 muestras.Para la medición del $\mathrm{pH}$ se pesó $5,0 \mathrm{~g}$ de pasta dental en un vaso de $100 \mathrm{~mL}$. Fue disuelto con agua destilada y aforado a $100 \mathrm{~mL}$. Se midió el $\mathrm{pH}$ con pHmeter.

Los resultados obtenidos en el laboratorio y el rotulado de las pastas dentales se procesaron siguiendo un patrón de tabulación automatizado con el soporte del paquete estadístico SPSS 22. En el análisis estadístico se empleó la prueba $t$ de Student de una muestra (para seis tipos de pastas) y la prueba de Wilcoxon para una muestra (para dos tipos de pasta). Para el estudio se considera como un valor poblacional las ppm del rotulado. No se está calculando la diferencia promedio en el grupo completo de pastas ya que, debido a los valores extremos entre las pastas, el promedio global se vería afectado como medida de resumen. El valor alfa fue de 0,05 .

Para la creación de la base de datos, tablas de frecuencia se utilizó el programa Microsoft Excel. Se cumplió con las normas éticas exigidas internacionalmente para estudios observacionales. Se siguieron los lineamientos de bioseguridad vigentes (8). El proyecto fue revisado y aceptado por la Escuela Académica Profesional de Odontología de la Universidad Priva- 
da Norbert Wiener, Lima, Perú. No se recogieron datos de personas para el estudio.

\section{RESULTADOS}

Se encontró que tres de pastas dentales presentaron un promedio menor al valor mostrado en el rotulado (Colgate de 1100 ppm, Farma Dent de 452 ppm, Dentito de $550 \mathrm{ppm}$ ), dos pastas dentales presentaron una mediana menor al valor mostrado en el rotulado (Colgate de 1100 ppm, Oral B de 500 ppm), dos pastas dentales presentaron un promedio mayor al valor mostrado en el rotulado (Vitis de 1000 ppm, Aqua Fresh de $1150 \mathrm{ppm}$ ), y, una pasta dental, si bien presentó un promedio mayor al presentado en el rotulado, no fue significativo como en los anteriores casos (Aqua fresh de $500 \mathrm{ppm}$ ).

En la tabla 1 se presenta la diferencia entre el ion flúor rotulado-encontrado y el $\mathrm{pH}$ de las pastas dentales.

\section{DISCUSIÓN}

Báez et al., encontraron que el 47\% (8 de 17) de cremas dentales presentaron valores entre el $5 \%$ y $15 \%$ por debajo del valor esperado por el estudio (9). En la presente investigación el 62,5\% (5 de 8) pastas dentales presentaron valores inferiores con respecto al rotulado. Según Cury et al., la mayoría de las pastas dentales estudiadas $(84,4 \%)$ coincide con lo indicado en el rotulado (10). En el presente estudio se halló diferencia significativa en 7 de 8 pastas dentales ( 2 en exceso y 5 en déficit).

Báez et al., utilizaron un electrodo específico de flúor Orion 9409-BN; Thermo Scientific Orion, Estados Unidos (9), mientras que Cury et al., utilizaron Orion model 96-09, Orion Research, Cambridge, MA, USA (10).

Tabla 1. Diferencia entre rotulado y encontrado en marcas de pastas dentales pediátricas

\begin{tabular}{|c|c|c|c|c|c|c|c|}
\hline \multirow[t]{2}{*}{$\begin{array}{l}\text { Tipos de Pastas dentales de } \\
\text { niños }\end{array}$} & \multicolumn{3}{|c|}{ PPM } & \multicolumn{2}{|c|}{$\begin{array}{l}\text { Prueba utilizada (va- } \\
\text { lor } p \text { ) }\end{array}$} & \multirow[b]{2}{*}{ Mediana } & \multirow[b]{2}{*}{ pH } \\
\hline & $\begin{array}{l}\text { Rotulado } \\
\text { (Ro) }\end{array}$ & $\begin{array}{l}\text { Promedio } \\
\text { encontrado } \\
\text { (E) }\end{array}$ & $\begin{array}{c}\text { Diferencia } \\
\text { (Ro-E)* }\end{array}$ & $\begin{array}{c}\text { Prueba } T \\
\text { para una } \\
\text { muestra }\end{array}$ & $\begin{array}{c}\text { Prueba } \\
\text { Wilcoxon } \\
\text { para una } \\
\text { muestra }\end{array}$ & & \\
\hline Colgate & 1100 & 974,3 & $-119,8$ & & 0,028 & $980,2 * *$ & 5,92 \\
\hline Colgate & 500 & 465,8 & $-34,2$ & 0,019 & & & 5,5 \\
\hline Oral B. & 500 & 434,19 & $-57,05$ & & 0,028 & $442,95 * *$ & 5,5 \\
\hline Farma Dent & 452 & 415,73 & $-36,27$ & 0,000 & & & 6 \\
\hline Dentito & 550 & 29,9 & -520.1 & 0,000 & & & 6 \\
\hline Aqua fresh & 500 & 541,67 & 41,67 & $0,151 * * *$ & & & 5,5 \\
\hline Vitis & 1000 & 1141,85 & 141,85 & 0,000 & & & 5,5 \\
\hline Aqua Fresh & 1150 & 1262,82 & 112,82 & 0,000 & & & 6 \\
\hline
\end{tabular}

*Valores positivos: sobrepasa las ppm en base al rotulado/Valores negativos: menor cantidad de ppm en base al rotulado 
Salvatierra encontró que las 7 pastas que analizó tuvieron valores por debajo de lo rotulado (7).

En base a las pastas analizadas por ambos estudios (con la misma concentración en el rotulado), en la marca Oral B, cuyo rotulado menciona 500 ppm, encontró un promedio de 242,25 ppm; el presente estudio en Oral B halló 434,19 ppm. Con respecto a la marca Colgate, cuyo rotulado menciona $500 \mathrm{ppm}$, encontró un promedio $376,96 \mathrm{ppm}$; el presente estudio en Colgate halló 465,8 de flúor. Con respecto a la marca Dentito, cuyo rotulado menciona $550 \mathrm{ppm}$, encontró un promedio $21,82 \mathrm{ppm}$; el presente estudio halló 29,9 ppm. Con respecto a la marca Vitis, cuyo rotulado menciona $1000 \mathrm{ppm}$, encontró un promedio 462,20 ppm; el presente estudio en Vitis halló $1141,85 \mathrm{ppm}$. Salvatierra encontró que los valores de $\mathrm{pH}$ de las pastas dentales estuvieron entre 6,24 y 6,74 (7). La presente investigación encontró una concentración de 5,5 siendo el mayor de 6; Salvatierra utilizó el mismo procedimiento y técnica que del presente estudio (7).

Giacaman et al., encontraron que 8 de 12 pastas dentales presentaron una coincidencia en la concentración de flúor con lo mencionado por el fabricante (11). En base a las pastas analizadas por ambos estudios (con la misma concentración en el rotulado), la mencionada investigación en la marca Colgate, con un rotulado de 1 100, encontró una concentración de flúor mayor a lo mencionado por el fabricante; en el presente estudio, se encontró una cantidad menor en la marca Colgate. Con respecto a la marca Oral B, con un rotulado de 500, encontró una concentración de flúor menor a lo mencionado por el fabricante; el presente estudio encontró el mismo resultado en la marca Oral B. Giacaman et al., utilizaron un electrodo específico de flúor Orion modelo 96-09- Orion Investigación, Cambridge, MA, USA (11).

No se contó con las especificaciones técnicas de la prueba de identificación de flúor en las pastas dentales estudiadas; por ser técnicas analíticas consideradas norma técnica propia. Dicha información es conocida solo por los respectivos fabricantes y autoridades competentes. No se tomó en cuenta otras variables como interacciones químicas de ingredientes inactivos, etc. Además, la muestra fue elegida por conveniencia.

Si bien la evidencia $(12,13)$ indica que el uso en menores debe ser como mínimo de 1000 ppm, es posi- ble que nuevos datos del uso de pastas dentales con menos de 1000 ppm, podrían cambiar esta dirección; para esto, la fabricación de pastas con menores concentraciones no debería ser descartada todavía (14). Los estudios futuros sobre comparación de eficacia de pastas con diferentes concentraciones, de preferencia en forma directa, deberían confirmar analíticamente, a priori, las concentraciones de flúor de las pastas a utilizar en el estudio.

Recomendamos que la autoridad correspondiente analice con regularidad las especificaciones técnicas de las pastas dentales (concentración de flúor y de su $\mathrm{pH})$.

Según los resultados obtenidos, concluimos que se encuentra diferencia significativa entre lo indicado en el rotulado y lo encontrado en la cantidad de flúor en 7 de 8 tipos de las pastas estudiadas; de las 7 pastas, cinco tuvieron diferencias menores y dos mayores.

\section{Correspondencia:}

César Arellano-Sacramento

Correo electrónico: cesar.arellano@uwiener.edu.pe

Conflictos de interés: Los autores declaran no presentar conflictos de intereses en relación con la preparación y publicación de este artículo.

Fuente de financiamiento: Este trabajo de investigación no ha recibido ningún tipo de apoyo financiero específico de instituciones públicas, privadas o sin ánimo de lucro.

\section{REFERENCIAS BIBLIOGRÁFICAS}

1. Martínez-Pabón MC, Galvis-Pareja DA, Builes-Sánchez ÁP, García-Ortega DA, Cañas-Londoño LT, Arango-Arango MI. The use of fluoride dentifrices in children: conceptual bases in a confusing context. A topic review. Rev Fac Odontol Univ Antioq. 2017; 29 (1):187-210.

2. Andina Agencia peruana de Noticias. Minsa: $85 \%$ de niños menores de 11 años tiene caries dental por inadecuada higiene bucal. Lima: ANDINA; 2017. (Citado el 25 de noviembre del 2017) Disponible en:

https://andina.pe/agencia/noticia-minsa-85-ni- 
nos-menores-11-anos-tiene-caries-inadecuada-higiene-bucal-690873.aspx

3. Ministerio de Salud. Norma Técnica Sanitaria para la Adición de Fluoruros en Cremas Dentales, Enjuagatorios y otros productos utilizados en la Higiene Bucal. RM. No 454-2001 SA/DM. Lima: Ministerio de Salud; 2001. (Citado el 25 de noviembre del 2017) Disponible en: http://www. digemid.minsa.gob.pe/UpLoad/UpLoaded/PDF/ RESOLUCIONMINISTERIALN454-2001-SA_ DM.pdf

4. Vallejos-Ragas R, Tineo-Tueros P. Administración de fluoruros en salud pública en el Perú. Debilidades y obstáculos. Rev Estomatol Herediana. 2015;25(1):78-83.

5. Ministerio de Salud. Decreto Supremo No 0162011/SA. Aprueban Reglamento para el Registro, Control y Vigilancia Sanitaria de Productos Farmacéuticos, Dispositivos Médicos y Productos Sanitarios. Lima: Ministerio de Salud; 2011. (Citado el 25 de noviembre del 2017) Disponible en: http://www.digemid.minsa.gob.pe/UpLoad/ UpLoaded/PDF/DS016-2011-MINSA.pdf

6. Instituto Nacional de Defensa de la Competencia y de la Protección de la Propiedad Intelectual. Marca de Producto. Lima: Instituto Nacional de Defensa de la Competencia y de la Protección de la Propiedad Intelectual; 2018.Citado el 15 de junio del 2018) Disponible en:

https://www.indecopi.gob.pe/web/signos-distintivos/marca-de-producto

7. Salvatierra D. Evaluación de la concentración de fluoruros contenido en los dentífricos bucales fluoradas para niños comercializados en el distrito de Trujillo, 2013. Tesis de Grado. Trujillo, Perú: Universidad Nacional de Trujillo; 2014.
8. Instituto Nacional de Salud. Manual de bioseguridad en laboratorios de ensayo, biomédicos y clínicos. Lima: Instituto Nacional de Salud;2005. (Citado el 15 de junio del 2018) Disponible en: http://www.ins.gob.pe/repositorioaps/0/0/jer/-1/ Manual\%20de\%20bioseguridad\%20-\%20INS. pdf

9. Báez-Quintero LC, Botazzo-Delbem AC, Nagata ME, Pessan JP. Concentración de flúor en cremas dentales y enjuagues bucales para niños vendidos en Bogotá, Colombia. Rev Nac Odontol. 2016;23(12):41-48

10. Cury JA, Oliveira MJL, Martins CCastro, Tenuta LMA, Paiva SM. Available Fluoride in Toothpastes Used by Brazilian Children. Braz Dent J. 2010; 21(5): 396-400.

11. Giacaman RA, Carrera CA, Muñoz-Sandoval C, Fernandez C, Cury JA. Fluoride content in toothpastes commercialized for children in Chile and discussion on professional recommendations of use. Int J Paediatr Dent. 2013;23(2):77-83.

12. Walsh T, Worthington H, Glenny A, Appelbe P, Marinho V, Shi X. Fluoride toothpastes of different concentrations for preventing dental caries in children and adolescents. Cochrane Database Syst Rev. 2010;1:CD007868. doi: 10.1002/14651858. CD007868.pub2

13. Ministerio de Salud. Guía de práctica clínica para la prevención, diagnóstico y tratamiento de la caries dental en niñas y niños. Lima: Ministerio de Salud; 2017.

14. Elías PMC, Arellano SC, Tello MPG. Odontología para Bebés. Fundamentos teóricos y prácticos para el clínico. Lima: Savia; 2016.

Recibido: 07-08-2018

Aceptado: 20-09-2019 